\title{
Beyond the $t$-channel Approximation:
} Next-to-Leading Order QCD Corrections to Electroweak Higgs Boson Plus Three Jet Production at the LHC

\section{Francisco Campanario}

Theory Division, IFIC, University of Valencia-CSIC

E-46100 Paterna, Valencia, Spain

E-mail: francisco.campanariodific.uv.es

\section{Terrance M. Figy*}

School of Physics and Astronomy, The University of Manchester

Manchester, M13 9PL, United Kingdom

E-mail: Terrance.Figy@hep.manchester.ac.uk

\section{Simon Plätzer}

Theory Group, DESY

D-22607 Hamburg, Germany

E-mail:simon.plaetzer@desy.de

\section{Malin Sjödahl}

Department of Astronomy and Theoretical Physics, Lund University

SE-22362 Lund, Sweden

malin.sjodahldthep.lu.se

In this talk we discuss the implementation of the full next-to-leading order QCD corrections to electroweak Higgs boson plus three jet production at the LHC within the Matchbox framework of the Herwig++ event generator. We also present numerical results for integrated cross sections and kinematic distributions.

Loops and Legs in Quantum Field Theory - LL 2014,

27 April - 2 May 2014

Weimar, Germany

\footnotetext{
* Speaker.
} 


\section{Introduction}

The existence of a new boson with a mass in the range of $125-126 \mathrm{GeV}$, with a spin most likely equal to zero and with even parity has been confirmed with increasing confidence in recent reports by the ATLAS and CMS Collaborations [1, 2, 3, 4]. Furthermore, the new particle exhibits production and decay rates similar to a Standard Model (SM) Higgs boson [5, 6, 7, 8, 9, 10].

Higgs boson production via vector boson fusion (VBF), i.e., the $t$-channel $\mathscr{O}\left(\alpha_{Q E D}^{3}\right)$ merging of two weak bosons in the reaction $q q \rightarrow q q H$, is an essential channel at the LHC for constraining Higgs boson couplings to gauge bosons and fermions. In the current experimental data from the LHC, the ATLAS Collaboration finds $3 \sigma$ evidence [9] for Higgs boson production via VBF and the CMS Collaboration finds $1.3 \sigma$ evidence [11].

For this process the observation of two forward tagging jets is crucial for the reduction of background. Requiring, in addition, that there is no extra radiation within the rapidity gap between the forward tagging jets $[12,13,14]$, i.e., imposing a central jet veto (CJV), suppresses standard QCD backgrounds, as well as Higgs production via gluon-gluon fusion in association with two jets (GF $H j j)$ [12, 13, 14].

To exploit the CJV strategy for Higgs boson coupling measurements, it is therefore necessary to know the reduction due to the CJV accurately. Thus it is of interest to calculate the ratio of Higgs boson plus three jet (EW $\mathrm{H} \mathrm{j}$ j j) production (where the third jet is required to be between the two tagging jets) to the inclusive Higgs boson plus two jet (EW $H$ j j ) cross section.

Recently the competing GF $H j j j$ has been computed within the heavy top effective theory approximation to next-to-leading order (NLO) in perturbative QCD [15]. The heavy top effective theory approximation for $H j j(j)$ has been validated against $H j j(j)$ amplitudes where the top mass dependence has been kept in Refs. [16, 17].

Approximated results at NLO QCD for EW VBF $H j j j$ production were presented sometime ago in $[18,19]$. There, the $t$-channel approximation was used and additionally, the inclusion of pentagon and hexagon one-loop Feynman diagram topologies (Figure 1, last two diagrams) and the corresponding real emission contributions were omitted and estimated to contribute at the permille level. Recently, parton-shower effects on EW VBF $H j j j$ were investigated in Ref. [20] within the $t$-channel approximation. In view of the relevance to the determination of Higgs boson couplings, we will present results from [21,22], where those approximations are lifted, and the full NLO QCD corrections to the $\mathscr{O}\left(\alpha_{s} \alpha_{E W}^{3}\right)$ production of a Higgs boson in association of three jets is calculated for the first time. In this proceedings, we show results for the inclusive sample and leave for future work a thorough comparison with the VBF approximation.

The remainder of this proceedings is organized as follows: Details of the NLO calculation are presented in Section 2. Numerical results and conclusions are shown in Section 3 and in Section 4, respectively.

\section{Calculational Details}

For the leading order (LO) $2 \rightarrow H+n(n=2,3,4)$ parton matrix elements, we employ the builtin spinor helicity library of the Matchbox module in the Herwig++ event generator $[23,24]$ to construct the full amplitude from hadronic currents [25]. The LO $2 \rightarrow H+n(n=2,3,4)$ parton matrix elements were also cross checked against Sherpa [26, 27], VBFNLO [28, 29, 30], and 
Hawk [31, 32]. The Catani-Seymour dipole subtraction terms [33] are generated automatically by the Matchbox module [25], and for efficient generation of phase space points, we utilize a diagram-based multichannel phase space sampler [25].

The computation of the interference of the virtual one-loop amplitude with Born amplitudes, is calculated with the aid of the helicity amplitude technique described in Ref. [34], using the program described in Ref. [35], which also provides an independent version of the Born amplitudes, providing a valuable internal consistency check of our implementation. A representative set of oneloop Feynman diagram topologies that contribute to the virtual corrections are depicted in Figure 1. To evaluate the one-loop tensor coefficients, we use the Passarino-Veltman approach [36] up to four-point functions, and the Denner-Dittmaier scheme [37], following the layout and notation of [35], to numerically evaluate the five and six point coefficients. The one-loop scalar integrals are in turn evaluated using the program OneLOop [38]. Complex masses and finite width effects in gauge boson propagators are calculated in the complex mass scheme [39, 40]. The resulting one-loop amplitudes for specific phase space points have been cross checked against GoSam [41].
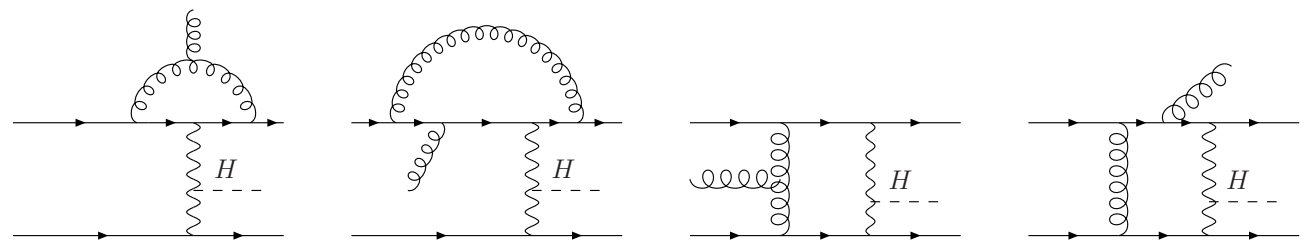

Figure 1: A representative selection of one-loop Feynman diagram topologies for EW $H j j j$ production.

The numerical stability of our code, is tested by employing a Ward identity check at each phase space point and each Feynman diagram [35] - at the cost of a small increase in computing time. Upon failure of the Ward identity check, the amplitudes of the gauge related topology are set to zero. The failure rate is at the per-mille level and hence under control. This method has also been successfully applied in other scattering processes with $2 \rightarrow 4$ kinematics $[42,43]$, however, in the work presented here, the method is applied to a process which involves loop propagators with complex masses for the first time.

The color structure associated with the computation of color correlated Born matrix elements has been performed by Colorfull [44] and cross checked against ColorMath [45]. As a further check of our framework, we have implemented the corresponding calculation of electroweak $H j j$ production and, subsequently, performed cross checks against Hawk [31, 32] and VBFNLO [28, 29, 30].

We refer to our implementation of the NLO corrections in perturbative QCD for electroweak Higgs boson plus two and three jet production in the Mat chbox framework as HJet +++ .

\section{Results}

In this section, we present results for a LHC of center-of-mass energy $\sqrt{s}=14 \mathrm{TeV}$. Here, we do not include parton shower and harmonization effects in our simulations. Instead the matrix element partons are recombined into jets according to the anti- $k_{T}$ algorithm [46] using Fast Jet [47] with $D=0.4$ and $E$-scheme recombination. We select events with at least three jets having 


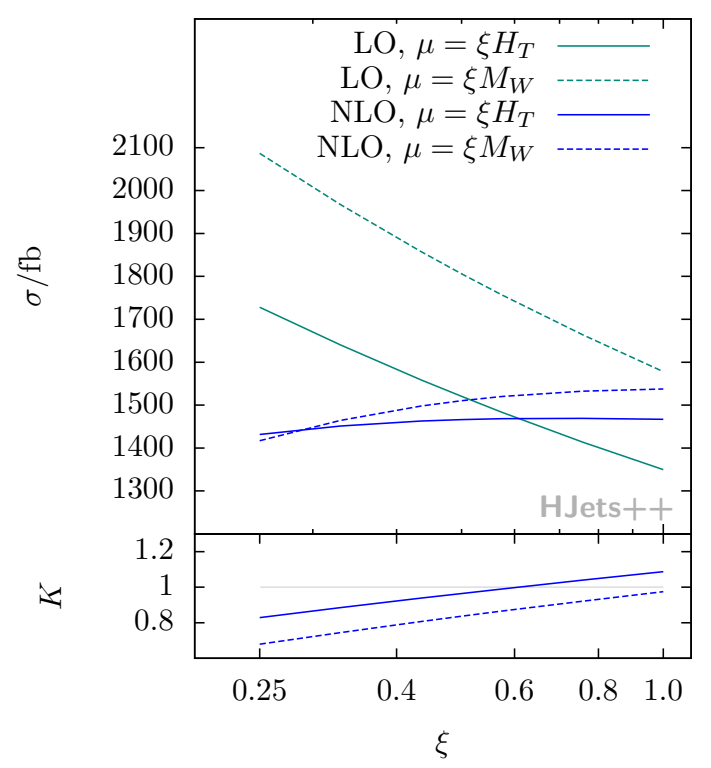

Figure 2: The $H j j j$ inclusive total cross section (in fb) at LO (cyan) and at NLO (blue) for the scale choices, $\mu=\xi M_{W}$ (dashed) and $\mu=\xi H_{T}$ (solid). The lower panel displays the $K$-factor, $K=\sigma_{N L O} / \sigma_{L O} \mathrm{f}$ or $\mu=\xi M_{W}$ (dashed) and $\mu=\xi H_{T}$ (solid).

transverse momentum $p_{T, j} \geq 20 \mathrm{GeV}$ and rapidity $\left|y_{j}\right| \leq 4.5$ and order the jets according to their transverse momentum.

We use the CT10 [48] parton distribution functions with $\alpha_{s}\left(M_{Z}\right)=0.118$ at NLO, and the CTEQ6L1 set [49] with $\alpha_{s}\left(M_{Z}\right)=0.130$ at LO. We use the five-flavor scheme for the running of $\alpha_{s}$. We choose $m_{Z}=91.188 \mathrm{GeV}, m_{W}=80.419002 \mathrm{GeV}, m_{H}=125 \mathrm{GeV}$ and $G_{F}=1.16637 \times$ $10^{-5} \mathrm{GeV}^{-2}$ as electroweak input parameters and derive the weak mixing angle $\sin \theta_{W}$ and $\alpha_{Q E D}$ from SM tree level relations. All fermion masses (except the top quark) are set to zero and the CKM matrix is taken to be diagonal. Widths are fixed to the following values: $\Gamma_{W}=2.0476 \mathrm{GeV}$ and $\Gamma_{Z}=2.4414 \mathrm{GeV}$.

In Figure 2, we show the LO and NLO total cross-sections for inclusive cuts for different values of the factorization scale $\left(\mu_{F}\right)$ and renormalization scale $\left(\mu_{R}\right)$, varied around the central scale, $\mu$ for two different scale choices, $M_{W} / 2$, and the scalar sum of the jet transverse momenta, $H_{T} / 2$ with $H_{T}=\sum_{j} p_{T, j}$. In general, we find - as expected - a decreased scale dependence in the NLO results. We also note that the central values for the various scale choices are closer to each other at NLO. The uncertainties, obtained by varying the central value a factor two up and down, are around $25 \%(28 \%)$ at $\mathrm{LO}$ and $2 \%(8 \%)$ at NLO using $H_{T} / 2\left(M_{W} / 2\right)$ as scale choice. For the scale choice $\mu=H_{T} / 2$, we obtained $\sigma_{L O}=1520(8)_{-171}^{+208} \mathrm{fb}$ and $\sigma_{N L O}=1466(17)_{-35}^{+1} \mathrm{fb}$. Studying differential distributions, we find that these generally vary less using the scalar transverse momentum sum choice, used from now on.

On the left-hand side of Figure 3, the differential distribution of the third jet, (i.e., the jet which would be vetoed in a CJV analysis), is shown. Here we find large $K$ factors in the high energy tail of the transverse momentum distribution. However, when VBF cuts ${ }^{1}$ are imposed the

\footnotetext{
${ }^{1}$ For the VBF cuts we have chosen to include the following cuts in addition to the inclusive cuts described in the
} 

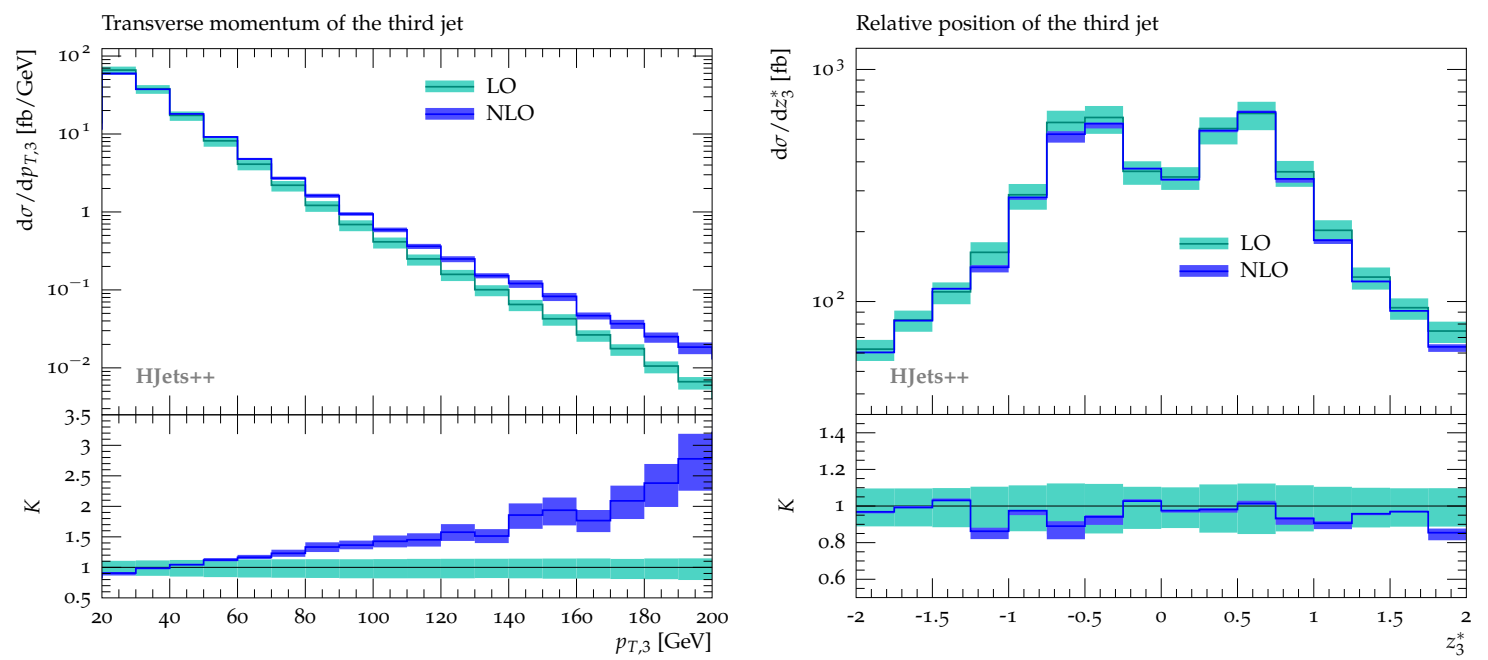

Figure 3: Differential cross section and $K$ factor for the $p_{T}$ of the third hardest jet (left) and the normalized centralized rapidity distribution of the third jet w.r.t. the tagging jets (right). Cuts are described in the text. The bands correspond to varying $\mu_{F}=\mu_{R}$ by factors $1 / 2$ and 2 around the central value $H_{T} / 2$.
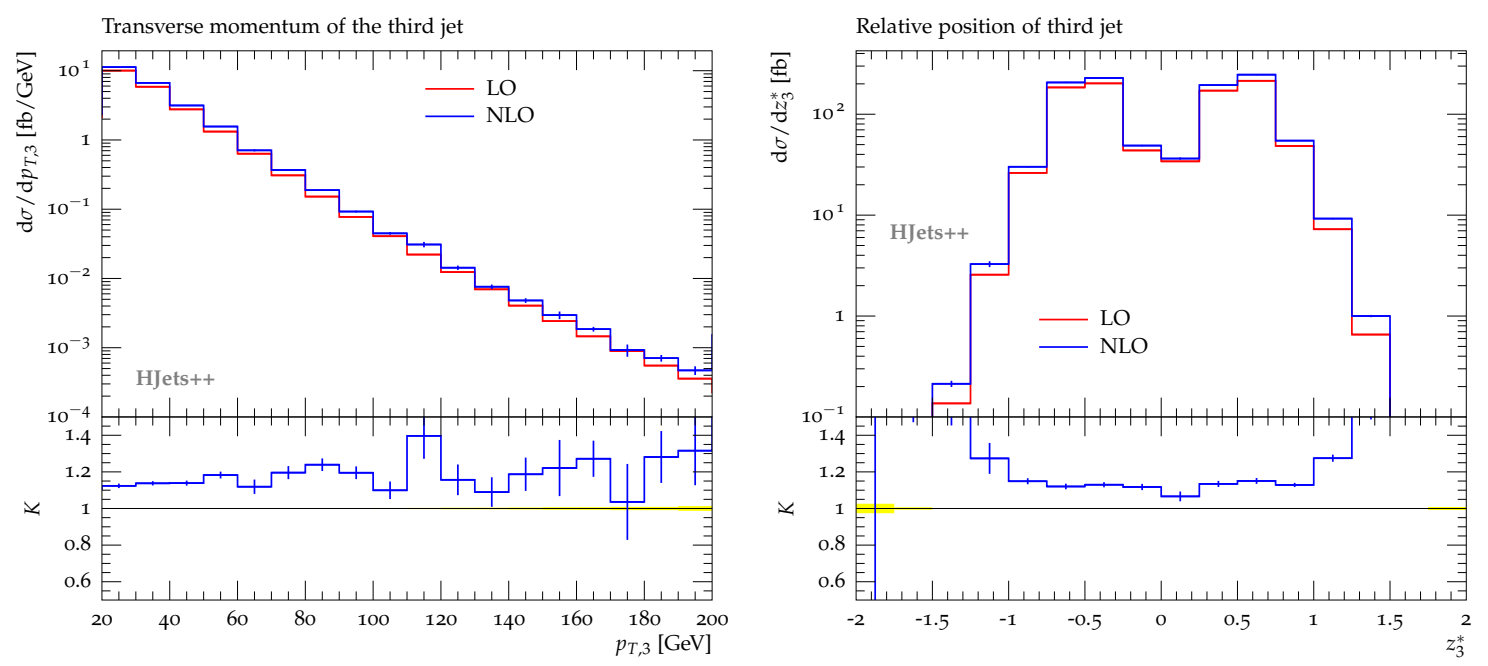

Figure 4: Differential cross section and $K$ factor for the $p_{T}$ of the third hardest jet (left) and the normalized centralized rapidity distribution of the third jet w.r.t. the tagging jets (right) with $\mu_{R}=\mu_{F}=H_{T}$. Beyond the inclusive cuts described in the text, we include the set of VBF cuts: $m_{12}=\sqrt{\left(p_{1}+p_{2}\right)^{2}}>600 \mathrm{GeV}$ and $\left|\Delta y_{12}\right|=\left|y_{1}-y_{2}\right|>4.0$.

$K$ factor is almost flat as a function of the transverse momentum of the third jet (see the left-hand side of Figure 4). On the right-hand side of Figure 3, we show the normalized centralized rapidity distribution of the third jet w.r.t. the tagging jets, $z_{3}^{*}=\left(y_{3}-\frac{1}{2}\left(y_{1}+y_{2}\right)\right) /\left(y_{1}-y_{2}\right)$. This variable, showing how the third jet tends to accompany one of the leading jets appearing at $1 / 2$ and $-1 / 2$ respectively, beautifully displays the VBF nature present in the process.

This effect is even more pronounced when VBF cuts are applied (see Figure 4), and should be contrasted with the gluon fusion production mechanism where QCD radiation in the rapidity gap

$\overline{\text { main text : } m_{12}=\sqrt{\left(p_{1}+p_{2}\right)^{2}}>600 \mathrm{GeV} \text { and }}\left|\Delta y_{12}\right|=\left|y_{1}-y_{2}\right|>4.0$ 
region between the leading two jets is much more common $[50,51,16,15]$.

\section{Conclusions}

In this proceedings, we have presented complete results at NLO QCD for electroweak Higgs boson production in association with three jets. We have found that the NLO corrections to the total inclusive cross section are moderate for inclusive cuts using the scale choice of $H_{T} / 2$. However, for the scale choice of $M_{W} / 2$, the NLO corrections can be more significant. The scale uncertainty decreases from around $25 \%(28 \%)$ at LO down to about $2 \%(8 \%)$ at NLO using the scale choice of $H_{T} / 2\left(M_{W} / 2\right)$. We have also presented numerical results showing the impact of VBF selection cuts on the transverse momentum of the third jet, $p_{T, 3}$, and its relative position w.r.t. the two leading jets, $z_{3}^{*}$.

\section{Acknowledgments}

We are grateful to Ken Arnold for contributions at an early stage of this project and to Mike Seymour and Jeff Forshaw for valuable discussions on the subject. F.C. is funded by a Marie Curie fellowship (PIEF-GA-2011- 298960) and partially by MINECO (FPA2011-23596) and by LHCPhenonet (PITN-GA-2010-264564). T.F. would like to thank the North American Foundation for The University of Manchester and George Rigg for their financial support. S.P. has been supported in part by the Helmholtz Alliance "Physics at the Terascale" and M.S. was supported by the Swedish Research Council, contract number 621-2010-3326. Numerical computations presented in this proceedings were performed via PhenoGrid using GridPP infrastructure and via the DESY Computing Cluster called Bird.

\section{References}

[1] ATLAS Collaboration Collaboration, G. Aad et al., Observation of a new particle in the search for the Standard Model Higgs boson with the ATLAS detector at the LHC, Phys. Lett. B716 (2012) 1-29, [arXiv:1207.7214].

[2] S. Chatrchyan et al., Observation of a new boson at a mass of $125 \mathrm{GeV}$ with the CMS experiment at the LHC, Phys. Lett. B 716 (Jul, 2012) 30-61. 59 p.

[3] ATLAS Collaboration Collaboration, G. Aad et al., Evidence for the spin-0 nature of the Higgs boson using ATLAS data, arXiv: 1307.1432.

[4] Combination of standard model Higgs boson searches and measurements of the properties of the new boson with a mass near $125 \mathrm{GeV}$, Tech. Rep. CMS-PAS-HIG-13-005, CERN, Geneva, 2013.

[5] P. W. Higgs, Broken Symmetries and the Masses of Gauge Bosons, Phys. Rev. Lett. 13 (1964) 508-509.

[6] P. W. Higgs, Broken symmetries, massless particles and gauge fields, Phys. Lett. 12 (1964) 132-133.

[7] F. Englert and R. Brout, Broken Symmetry and the Mass of Gauge Vector Mesons, Phys. Rev. Lett. 13 (1964) 321-323.

[8] G. Guralnik, C. Hagen, and T. Kibble, Global Conservation Laws and Massless Particles, Phys. Rev. Lett. 13 (1964) 585-587.

[9] ATLAS Collaboration Collaboration, G. Aad et al., Measurements of Higgs boson production and couplings in diboson final states with the ATLAS detector at the LHC, arXiv: 1307.1427. 
[10] Properties of the observed higgs-like resonance using the diphoton channel, Tech. Rep. CMS-PAS-HIG-13-016, CERN, Geneva, 2013.

[11] Update of the search for the Standard Model Higgs boson decaying into WW in the vector boson fusion production channel, Tech. Rep. CMS-PAS-HIG-13-022, CERN, Geneva, 2013.

[12] V. D. Barger, R. Phillips, and D. Zeppenfeld, Mini - jet veto: A Tool for the heavy Higgs search at the LHC, Phys. Lett. B346 (1995) 106-114, [hep-ph/9412276].

[13] D. L. Rainwater, D. Zeppenfeld, and K. Hagiwara, Searching for $H \rightarrow \tau^{+} \tau^{-}$in weak boson fusion at the CERN LHC, Phys. Rev. D59 (1998) 014037, [hep-ph / 9808468 ].

[14] D. L. Rainwater and D. Zeppenfeld, Observing $H \rightarrow W^{*} W^{*} \rightarrow e^{ \pm} \mu \mp \not p_{T}$ in weak boson fusion with dual forward jet tagging at the CERN LHC, Phys. Rev. D60 (1999) 113004, [hep-ph/ 9906218 ].

[15] G. Cullen, H. van Deurzen, N. Greiner, G. Luisoni, P. Mastrolia, et al., NLO QCD corrections to Higgs boson production plus three jets in gluon fusion, Phys. Rev. Lett. 111 (2013) 131801, [arXiv:1307.4737].

[16] F. Campanario and M. Kubocz, Higgs boson production in association with three jets via gluon fusion at the LHC: Gluonic contributions, Phys. Rev. D88 (2013) 054021, [arXiv: 1306.1830 ].

[17] V. Del Duca, W. Kilgore, C. Oleari, C. Schmidt, and D. Zeppenfeld, Higgs + 2 jets via gluon fusion, Phys. Rev. Lett. 87 (2001) 122001, [hep-ph/ 0105129 ].

[18] T. Figy, V. Hankele, and D. Zeppenfeld, Next-to-leading order QCD corrections to Higgs plus three jet production in vector-boson fusion, JHEP 0802 (2008) 076, [arXiv: 0710 . 5621].

[19] T. M. Figy, NLO QCD corrections to the jet activity in Higgs boson production via vector-boson fusion, Ph.D. Thesis (2006).

[20] B. Jager, F. Schissler, and D. Zeppenfeld, Parton-shower effects on Higgs boson production via vector-boson fusion in association with three jets, arXiv:1405.6950.

[21] F. Campanario, T. M. Figy, S. Plätzer, and M. Sjödahl, Electroweak higgs boson plus three jet production at next-to-leading-order qcd, Phys. Rev. Lett. 111 (Nov, 2013) 211802.

[22] F. Campanario, T. M. Figy, S. Platzer, and M. Sjodahl, NLO QCD Corrections to Electroweak Higgs Boson Plus Three Jet Production at the LHC, PoS RADCOR2013 (2014) 042, [arXiv:1311.5455].

[23] M. Bähr et al., Herwig++ Physics and Manual, Eur. Phys. J. C58 (2008) 639-707, [arXiv:0803.0883].

[24] J. Bellm, S. Gieseke, D. Grellscheid, A. Papaefstathiou, S. Platzer, et al., Herwig++ 2.7 Release Note, arXiv: 1310.6877 .

[25] S. Platzer and S. Gieseke, Dipole Showers and Automated NLO Matching in Herwig++, Eur. Phys. J. C72 (2012) 2187, [arXiv:1109.6256].

[26] T. Gleisberg et al., SHERPA 1.alpha, a proof-of-concept version, JHEP 02 (2004) 056, [hep-ph/0311263].

[27] T. Gleisberg et al., Event generation with SHERPA 1.1, JHEP 02 (2009) 007, [arXiv: 0811.4622 ].

[28] K. Arnold, J. Bellm, G. Bozzi, M. Brieg, F. Campanario, et al., VBFNLO: A Parton Level Monte Carlo for Processes with Electroweak Bosons - Manual for Version 2.5.0, arXiv: 1107.4038. 
[29] K. Arnold, J. Bellm, G. Bozzi, F. Campanario, C. Englert, et al., Release Note - Vbfnlo-2.6.0, arXiv:1207.4975.

[30] J. Baglio, J. Bellm, F. Campanario, B. Feigl, J. Frank, et al., Release Note - VBFNLO 2.7.0, arXiv: 1404.3940.

[31] M. Ciccolini, A. Denner, and S. Dittmaier, Strong and electroweak corrections to the production of Higgs + 2jets via weak interactions at the LHC, Phys. Rev. Lett. 99 (2007) 161803, [arXiv:0707.0381].

[32] M. Ciccolini, A. Denner, and S. Dittmaier, Electroweak and QCD corrections to Higgs production via vector-boson fusion at the LHC, Phys. Rev. D77 (2008) 013002, [arXiv: 0710 . 4749].

[33] S. Catani and M.H. Seymour, A general algorithm for calculating jet cross sections in NLO QCD, Nucl. Phys. B485 (1997) 291-419, [hep-ph / 9605323$].$

[34] K. Hagiwara and D. Zeppenfeld, Amplitudes for Multiparton Processes Involving a Current at e+e-, e+- p, and Hadron Colliders, Nucl. Phys. B313 (1989) 560.

[35] F. Campanario, Towards $p p \rightarrow V V j j$ at NLO QCD: Bosonic contributions to triple vector boson production plus jet, JHEP 1110 (2011) 070, [arXiv: 1105 . 0920].

[36] G. Passarino and M. Veltman, One Loop Corrections for e+e-Annihilation Into mu+ mu- in the Weinberg Model, Nucl. Phys. B160 (1979) 151.

[37] A. Denner and S. Dittmaier, Reduction schemes for one-loop tensor integrals, Nucl. Phys. B734 (2006) 62-115, [hep-ph/0509141].

[38] A. van Hameren, OneLOop: For the evaluation of one-loop scalar functions, Comput. Phys. Commun. 182 (2011) 2427-2438, [arXiv: 1007 .4716].

[39] A. Denner and S. Dittmaier, The Complex-mass scheme for perturbative calculations with unstable particles, Nucl. Phys. Proc. Suppl. 160 (2006) 22-26, [hep-ph / 0605312$].$

[40] M. Nowakowski and A. Pilaftsis, On gauge invariance of Breit-Wigner propagators, Z. Phys. C60 (1993) 121-126, [hep-ph/9305321].

[41] G. Cullen, N. Greiner, G. Heinrich, G. Luisoni, P. Mastrolia, et al., Automated One-Loop Calculations with GoSam, Eur. Phys. J. C72 (2012) 1889, [arXiv:1111.2034].

[42] F. Campanario, C. Englert, M. Rauch, and D. Zeppenfeld, Precise predictions for $W \gamma \gamma+j e t$ production at hadron colliders, Phys. Lett. B704 (2011) 515-519.

[43] F. Campanario, M. Kerner, L. D. Ninh, and D. Zeppenfeld, WZ production in association with two jets at NLO in QCD, Phys. Rev. Lett. 111, 052003 (2013) [arXiv:1305.1623].

[44] M. Sjodahl, ColorFull - A C++ package for color space calculations, http://colorfull.hepforge.org/.

[45] M. Sjodahl, ColorMath - A package for color summed calculations in SU(Nc), Eur. Phys. J. C73 (2013) 2310, [arXiv:1211.2099].

[46] M. Cacciari, G. P. Salam, and G. Soyez, The Anti-k(t) jet clustering algorithm, JHEP 0804 (2008) 063, [arXiv:0802.1189].

[47] M. Cacciari, G. P. Salam, and G. Soyez, FastJet User Manual, Eur. Phys. J. C72 (2012) 1896, [arXiv:1111.6097].

[48] H.-L. Lai, M. Guzzi, J. Huston, Z. Li, P. M. Nadolsky, et al., New parton distributions for collider physics, Phys. Rev. D82 (2010) 074024, [arXiv: 1007.2241$].$ 
[49] J. Pumplin, D. Stump, J. Huston, H. Lai, P. M. Nadolsky, et al., New generation of parton distributions with uncertainties from global QCD analysis, JHEP 0207 (2002) 012, [hep-ph / 0201195 ].

[50] J. R. Forshaw and M. Sjodahl, Soft gluons in Higgs plus two jet production, JHEP 0709 (2007) 119, [arXiv:0705.1504].

[51] B. E. Cox, J. R. Forshaw, and A. D. Pilkington, Extracting Higgs boson couplings using a jet veto, Phys. Lett. B696 (2011) 87-91, [arXiv: 1006.0986$].$ 